ohoku J. exp. Med., 1968, 96, 327-331

\title{
Serum Heat-stable Alkaline Phosphatase and Placental Function
}

\author{
Ryuji Yamaguchi, Takeshi Yoshida, \\ Chuichi Kimura and Yoshinori Hatakeyama \\ Department of Obstetrics and Gynecology (Prof. K. Kushima), \\ Tohoku University School of Medicine, Sendai
}

\begin{abstract}
The pattern of serum heat-stable alkaline phosphatase (HSAP) of women in late pregnancy was examined and discussed in relation to the clinical course. Four types were recognized in the HSAP pattern. The unstable type was most closely related to the clinical course; that is, this type was observed in only 6 out of 79 cases of the normal group, whereas it was found in 12 out of 24 of the postdated group and 5 out of 10 of the toxemia group. The incidence of intrauterine fetal distress and of placental dysfunction syndrome was also highest in the unstable type. The significance of the unstable type was stressed as an alarm signal for fetal life in the third trimester of pregnancy.
\end{abstract}

Several studies on serum heat-stable alkaline phosphatase (HSAP) in pregnant women have been reported, and its value as an index of the placental function is now well recognized. ${ }^{1-5}$ In a previous report of ours, ${ }^{3}$ we have revealed that ISSAP pattern near term is most important for the evaluation of the placental function. The purpose of this paper is to describe the clinical significance of the unstable type of HSAP.

\section{Subjects and Method}

Sera obtained repeatedly from 113 cases of pregnant women near term were examined. As a rule, out-patients were examined once a week, while in-patients at least twice a week or every day. The serum was heated at $56^{\circ} \mathrm{C}$ for 30 minutes, and then the activity of HSAP was determined with a Cica PHOS kit and expressed in King-Armstrong units.

\section{Results}

In a previous report of ours ${ }^{3}$ we classified the pattern of HSAP into four types as shown in Fig. 1; that is, elevating, descending, plateau and unstable (or fluctuating) types. Table 1 shows the relation between the four types of HSAP and the clinical course of pregnancy. Among 36 cases of the elevating type, 33 cases belonged to the normal term group. On the other hand, only 6 out of 23 cases of the unstable type belonged to the normal term group. It is emphasized that 5 of 10 cases of toxemia were of the unstable type.

Received for publication, July $29,1968$. 


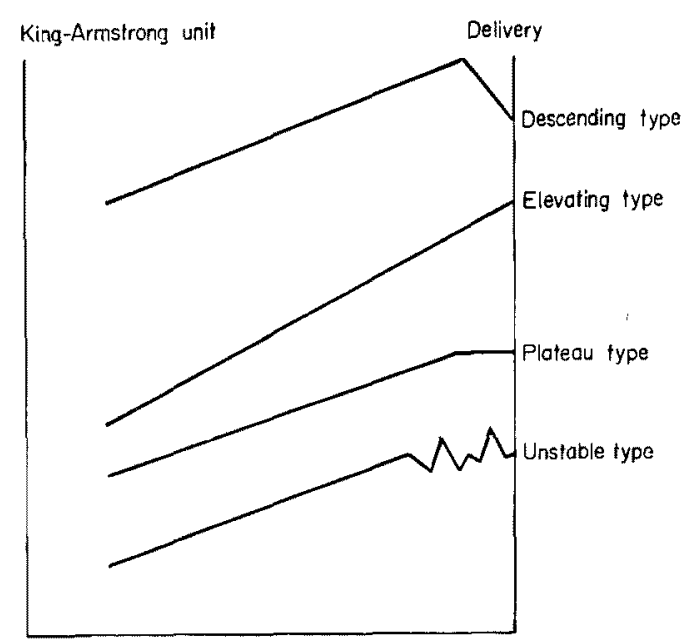

Fig. 1. Four types of HSAP pattern.

TABLE 1. Types of HSAP pattern and pregnancy group

\begin{tabular}{c|c|c|c|c}
\hline & Term group & Postdated group & Toxemia group & Total \\
\hline Elevating type & 33 & 3 & 0 & 36 \\
\hline Plateau type & 9 & 2 & 3 & 14 \\
\hline Descending type & 31 & 7 & 2 & 40 \\
\hline Unstable type & 6 & 12 & 5 & 23 \\
\hline Total & 79 & 24 & 10 & 113
\end{tabular}

Term group...... Within 2 weeks of calculated date of delivery.

Postdated group.....2 weeks or more extended beyond calculated date of delivery.

Toxemia group.......Mild and healed toxemias were excluded.

Clinical course of each type of HSAP is shown in Table 2. The rate of spontaneous delivery was highest in the descending type and lowest in the unstable type. Six of 9 cases of the placental dysfunction syndrome belonged to the unstable type. Intrauterine fetal distress and neonatal asphyxia were relatively frequent in the unstable type. These results suggest that the unstable type is not only specific to some extent for toxemia and prolonged pregnancy, but also an alarm signal for the fetus and mother.

The authors consider that the elevating type indicates an increasing activity of the placenta, and the unstable type a loss of its reserve capacity, and the other two types are intermediate forms between the elevating and unstable types. If this hypothesis is true, what is the reason for the fact that only a half of the post-dated group showed the unstable type? Two answers may be offered. One is that the definition of 'post-dated or prolonged pregnancy' over two weeks or more in reference to the calculated date of delivery is only a statistical or empirical one, 
TABLE 2. Types of HSAP and clinical course (113 cases)

\begin{tabular}{l|c|c|c|c|c|c}
\hline & Cases & $\begin{array}{c}\text { Spon- } \\
\text { taneous } \\
\text { delivery }\end{array}$ & $\begin{array}{c}\text { Obstetric } \\
\text { operation* }\end{array}$ & $\begin{array}{c}\text { Fetal } \\
\text { distress }\end{array}$ & $\begin{array}{c}\text { Neonatal } \\
\text { asphyxia }\end{array}$ & $\begin{array}{c}\text { Placental } \\
\text { dysfunction } \\
\text { syndrome }\end{array}$ \\
\hline Elevating type & 36 & 27 & 4 & 2 & 3 & 0 \\
\hline Plateau type & 14 & 11 & 3 & 1 & 1 & 1 \\
\hline Descending type & 40 & 38 & 12 & 4 & 3 & 2 \\
\hline Unstable type & $\mathbf{2 3}$ & 12 & 9 & 4 & 4 & 6
\end{tabular}

* Breech extraction was excluded from obstetric operation and neonatal asphyxia

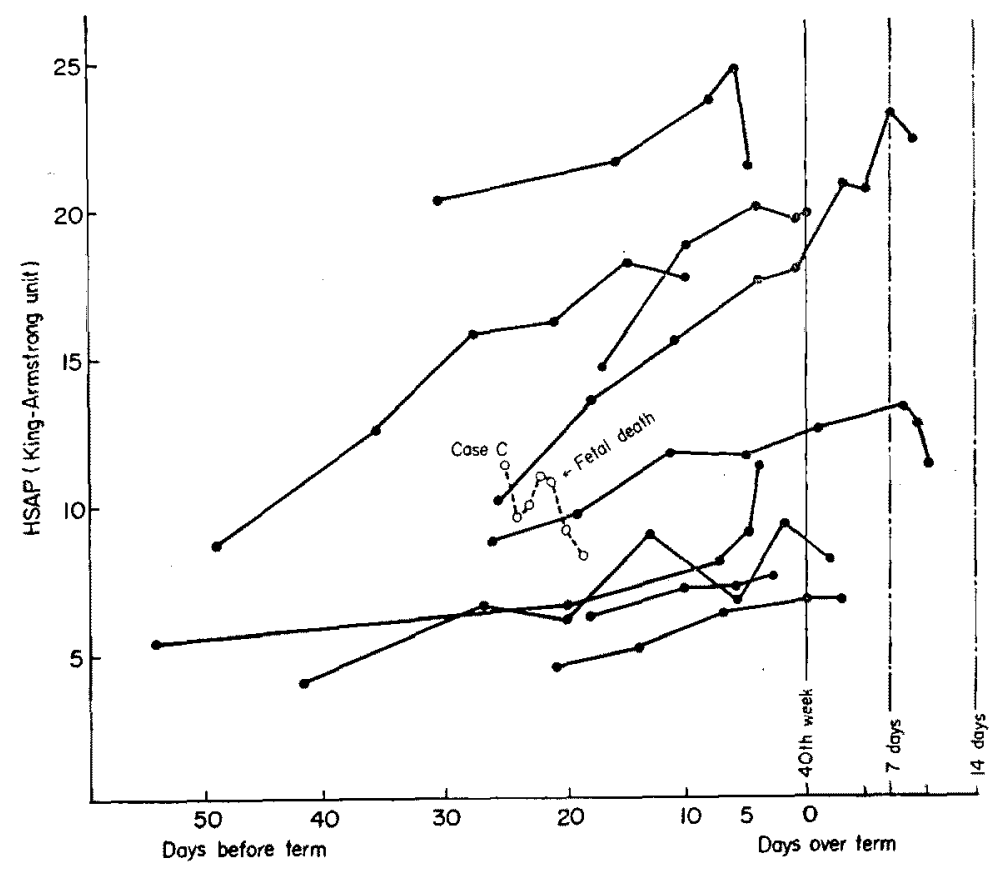

Fig. 2. HSAP pattern of normal term group.

and does not always coincide with biological 'postdate' of the placental activity. The other is that the apparent prolongation of the gestation for two weeks or more may be due to delayed ovulation and fertilization, or due to a slip of memory of the last menstruation. The cases $A$ and $B$ in Fig. 3, which showed the descending type and the elevating type respectively, are thought to be examples of the second category when interpreted from the appearance of vomiting and onset of fetal movement. These cases may support the view that as far as HSAP continues to elevate, the biological function of the placenta has not yet reached its maximum. Consequently, when HSAP continues to elevate, the induction of labor is not necessary even if the gestation exceeds the calculated date of delivery by two weeks 


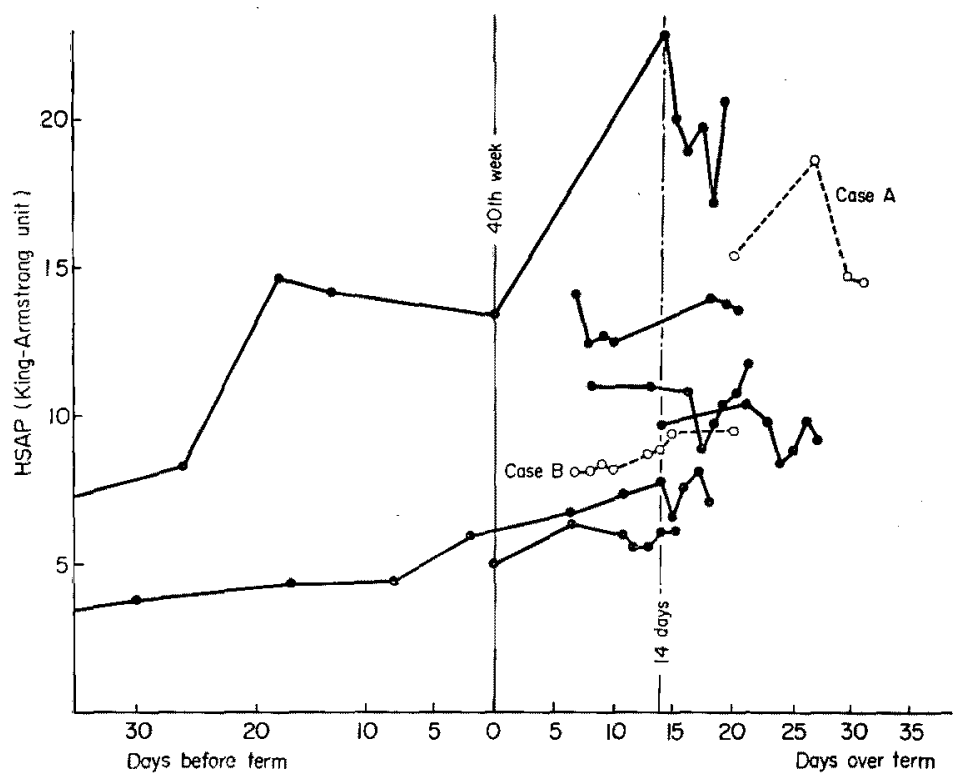

Fig. 3. HSAP pattern of postdated group.

or more. But when HSAP shows the unstable type, continuation of the gestation may exert an unfavorable influence upon the fetus regardless of the calculated date of delivery. In a case of severe toxemia with the unstable type, intrauterine fetal death occurred in the course of clinical observation (Case $C$ in Fig. 2). This suggests that the unstable type may precede fetal death as pointed out by Curzen and Morris. ${ }^{5}$

\section{Comment}

The present report describes further observations on the clinical significance of HSAP as pointed out in a previous report on 113 cases out of about 450 pregnant women. The HSAP pattern obtained by repeated examinations can be classified into four types, of which the unstable type has a clinical significance as an alarm signal for fetal life. A single examination is usually not reliable because of a wide variation in the normal value. The following schedule is recommended for the frequency of determination of HSAP pattern: once a week from the 37th to 39th week of gestation, twice or three times a week after the 39th week, and once every day or every other day in cases of severe toxemia of pregnancy, postdated pregnancy of over 2 weeks or other complicated pregnancies.

From our previous observation that HSAP was absent in the fetal blood and present only in the maternal blood after fetal death, it seems likely that HSAP indicates only placental, but not fetal, function. Therefore, the HSAP test as an index of the placental function is considered to be different from the urinary estriol test as an indicator of the fetoplacental function. 


\section{References}

1) Aoba, H., Hariu, Y. \& Yamaguchi, R. Serum heat-stable alkaline phosphatase in normal and abnormal pregnancy. Tohoku J. exp. Med., 1967, 91, 201-208.

2) Yamaguchi, R., Aoba, H. \& Hatakeyama, Y. Clinical observations of serum heatstable alkaline phosphatase. Sanfujinka no Sekai (Jap.), 1968, 20, 271-274.

3) Yamaguchi, R., Yoshida, T. \& Yazaki, S. On the clinical significance of serum heat. stable alkaline phosphatase as a test of placental function. Nippon Sanka-Fujinka Gakkai Zassi (Jap.), 1968, 20, 567-570.

4) Messer, R.H. Heat-stable alkaline phosphatase as an index of placental function. Amer. J. Obstet. Gynec., 1967, 98, 459-465.

5) Curzen, P. \& Morris, I. Heat-stable alkaline phosphatase in matemal serum. $J$. Obstet. Gynaec. Brit. Cwith., 1968, 75, 151-157. 